\title{
GERAÇÃO AUTOMATIZADA DE SÉRIES TEMPORAIS DE DADOS POSICIONAIS UTILIZANDO O IBGE-PPP
}

\section{Automated generation of time series of positional data using the IBGE-PPP}

\author{
Lécio Alves Nascimento ${ }^{1,2}$ \\ William Rodrigo Dal Poz ${ }^{2}$ \\ Afonso de Paula dos Santos ${ }^{2}$ \\ Krisley Xavier Soares de Freitas ${ }^{3}$
}

\begin{abstract}
${ }^{1}$ Instituto Federal de Educação, Ciência e Tecnologia do Norte de Minas Gerais (IFNMG) - Campus Araçuaí - MG, Brasil, email: lecio.nascimento@ifnmg.edu.br

2Universidade Federal de Viçosa - UFV - Departamento de Engenharia Civil, Setor de Engenharia de Agrimensura e Cartográfica, Programa de Pós-Graduação em Engenharia Civil da Universidade Federal de Viçosa - UFV, Viçosa - MG, Brasil, email: william.dalpoz@ufv.br; afonso.santos@ufv.br;
\end{abstract}

${ }^{3}$ Universidade Federal de Viçosa - UFV - Departamento de Engenharia Civil, Setor de Engenharia de Agrimensura e Cartográfica, Viçosa - MG, Brasil, email: krisley.freitas@ufv.br

\section{Resumo:}

Estudos que utilizam séries temporais são realizados em diversas áreas do conhecimento, como: economia, geofísica, geodésia etc. Dados específicos são associados a cada área supracitada, possibilitando efetuar análises sobre as séries, e avaliar as influências temporais de diversos fatores que interferem nos dados. No âmbito da geodésia espacial, vários estudos são desenvolvidos, de forma direta ou indireta, sobre séries temporais de dados posicionais (coordenadas), permitindo investigar a influência, por exemplo, da ionosfera, da troposfera, da geodinâmica etc. No entanto, a construção de uma série temporal posicional apresenta alguns empecilhos, que tornam esse procedimento moroso e, em muitos casos, inviável. Diante do exposto, esse trabalho tem como objetivo apresentar a metodologia desenvolvida e implementada no software RINEX EDITION, para a geração de séries temporais posicionais. Como resultados, geraram-se duas séries temporais posicionais (coordenadas cartesianas $\mathrm{X}, \mathrm{Y}$, $\mathrm{Z}$; elipsoidais $\varphi, \lambda, \mathrm{h}$ e as precisões de ambas), considerando um período de aproximadamente cinco anos e meio (5,5 anos), para duas estações da RBMC, utilizando o serviço de posicionamento IBGE-PPP. Uma comparação preliminar entre as séries das altitudes geométricas (h) e as séries de dados fluviométricos da Agencia Nacional de Águas (ANA) também foi efetuada, apresentando a influência da carga hidrológica na altitude geométrica.

Palavras-chave: IBGE-PPP, Séries Temporais, RINEX EDITION, Carga Hidrológica.

\begin{abstract}
:
Researches that use time series are done in several fields of studies, like economy, geophysics, geodesy, etc. Specific data are associated to each field of study, making possible to do analyses
\end{abstract}


over the series and evaluate the time influences of several factors that affect the data. Within the scope of spatial geodesy, many studies are developed, directly or indirectly, about time series of positional data (coordinates), allowing to explore the influence of: the ionosphere, the troposphere, the geodynamics, etc. However, the construction of a positioning time series shows some limitations, that make this process slow, and many times, impracticable. The goal of this paper is present the developed and implemented methodology in the RINEX EDITION software, to create positioning time series. As result, two positioning time series were created (Cartesian coordinates $\mathrm{X}, \mathrm{Y}, \mathrm{Z}$; ellipsoidal coordinates $\varphi, \lambda, \mathrm{h}$ and their precisions), considering a period of time about five and a half years (5,5 years), for two RBMC stations, using the online IBGE-PPP service. Also a preliminary comparison between the time series e of ellipsoidal height and of the hydrologic data of the National Agency of Water (ANA) was done, presenting the influence of the hydrologic load in the ellipsoidal height.

Keywords: IBGE-PPP, Time Series, RINEX EDITION, Hydrologic Load.

\section{Introdução}

Uma série temporal constitui-se em qualquer conjunto de dados ou variáveis ordenadas no tempo, ou qualquer outra variável que desempenhe a função de índice. A análise de séries temporais compõem diversas pesquisas, possibilitando revelar características de eventos que ocorrem no decorrer do tempo e tornando possível identificar os fatores que, de alguma maneira, regem o comportamento (Box, Jenkins e Reinsel 2008; Rosa 2008).

O impacto das análises de séries temporais em investigações científicas pode ser documentado, produzindo uma lista, contendo as diversas áreas em que podem surgir problemas importantes de séries temporais, como por exemplo, economia, epidemiologia, medicina, sismologia, geofísica, meteorologia, geodésia etc. (Shumway e Stoffer 2006). A cada área associa-se um ou mais tipos de dados específicos que compõem as séries, permitindo se efetuar análises diversas, de forma a se obter informações não somente sobre seu comportamento (tendencioso, cíclico ou sazonal), mas também sobre as influências temporais de alguns fatores que afetam os dados. Atualmente, uma gama de estudos com diversas finalidades são desenvolvidos, de forma direta ou indireta, sobre séries de dados de tipo posicional (coordenadas). Os dados de posição podem ser determinados utilizando algumas tecnologias que dão suporte ao posicionamento geodésico, como por exemplo, as tecnologias espaciais de posicionamento, com destaque ao GNSS (Global Navigation Satellite System).

Existem diversos métodos de posicionamento com GNSS, sendo possível construir séries temporais de coordenadas. Dentre as alternativas de métodos de posicionamento disponíveis pode-se citar o Posicionamento por Ponto Preciso (PPP) que, segundo Grinter e Roberts (2011) destaca-se em relação aos demais métodos por diversas maneiras, dentre elas, pela precisão alcançada usando apenas um único receptor. Ramos (2015) destaca que a acurácia e a praticidade proporcionada na aplicação do PPP, fez com que o método de processamento tornasse alvo de muitas investigações científicas.

Algumas dessas investigações requerem uma quantidade considerável de informações posicionais, exigindo que se determinem séries temporais, permitindo analisar efeitos cíclicos ou sazonais, como se pode observar em: Bueno (2007), que usa a análise de séries temporais para o monitoramento de estruturas com carga dinâmica; Rosa (2008), em que se utilizou das séries 
temporais estimadas com o PPP para estabelecer uma proposta de eliminação dos efeitos sazonais; Moreira (2010), a qual se utilizou do PPP para auxiliar sua pesquisa, que se constituiu na avaliação da altimetria por satélites na região amazônica; Silva e Monico (2012), que utilizam a análise de séries temporais para a caracterização de ruídos contidos nas séries temporais de coordenadas GNSS, utilizando soluções diárias disponibilizadas pelo SIRGAS-CON; Gu e Wang (2013), em que utiliza a análise de séries temporais para apresentar as vantagens de aplicações GNSS para o monitoramento de deformação na crosta com a finalidade de detecção de fortes terremotos; Collischonn et al. (2014), onde efetuou análise de séries temporais de forma a apresentar a correlação do posicionamento GNSS por ponto com a ionosfera e com índices de atividade solar no período de 2002 a 2011; Li (2015), que utiliza séries temporais no PPP em tempo real para monitoramento de terremotos e sistemas de alerta, dentre outros.

Diversos serviços de PPP são disponibilizados online, permitindo a construção de séries temporais posicionais. Na maioria dos estudos que demandam este tipo de série de dados é comum que se utilizem dados de redes ativas como a Rede Brasileira de Monitoramento Contínuo dos Sistemas GNSS (RBMC), que disponibiliza dados para longos períodos de tempo (aproximadamente 5 anos). Em suma, a construção de uma série pode ser subdividida em quatro etapas: download de dados GNSS; edição dos dados; processamento utilizando o PPP, e; extração das informações posicionais.

A construção de uma série temporal posicional, seguindo as quatro etapas supracitadas, apresentam alguns empecilhos tais como: conhecimento e utilização das linguagens de programação; demanda de tempo considerável para processamento dos dados GNSS utilizando os serviços de PPP; a morosidade na edição de dados GNSS e na extração das informações posicionais pós-processadas etc. Estes fatores tornam o procedimento de construção exaustivo e, em muitos casos, inviável. Nesse contexto, justifica-se a necessidade do desenvolvimento de uma metodologia automatizada que permita a construção de séries temporais de forma simples e objetiva.

Partindo-se das dificuldades supracitadas, ocasionadas na geração de uma série temporal de dados posicionais utilizando o PPP, o objetivo deste trabalho é apresentar a metodologia desenvolvida e implementada no software RINEX EDITION, para a geração automatizada de séries temporais posicionais. Para exemplificar a aplicação é apresentada a construção de uma série temporal posicional (coordenadas cartesianas $\mathrm{X}, \mathrm{Y}, \mathrm{Z}$; elipsoidais $\varphi, \lambda$, h e as precisões de ambas), para um período de aproximadamente cinco anos e meio (5,5 anos) para as estações NAUS (Manaus - AM) e SAGA (São Gabriel da Cachoeira - AM), pertencentes à RBMC. De posse dos dados gerados, efetuou-se uma comparação preliminar das séries temporais de altitudes geométricas (h) das estações selecionadas com as séries temporais de dados fluviométricos (cotas linimétricas), disponibilizados pela Agencia Nacional de Águas (ANA) em seu banco de dados HidroWeb (ANA 2016; SNIRH 2016) para as seguintes estações: MANAUS - ANA (código 14990000), situada nas proximidades da cidade de Manaus - AM e a estação SÃO GABRIEL DA CACHOEIRA - ANA (código 14320001), localizada nas proximidades do município de São Gabriel da Cachoeira - AM, no alto Rio Negro, de forma a verificar a influência da carga hidrológica na altitude geométrica para o período determinado (01/01/2010 a 13/08/2015).

A metodologia proposta é executada de forma automatizada onde cada etapa funciona de forma independente, proporcionando ao usuário subdividir a construção das séries posicionais da forma mais adequada. Outra consideração relevante consiste na minimização ou eliminação dos erros que podem ocorrer na etapa de edição manual dos dados bem como na etapa de extração das informações posicionais pós-processadas (coordenadas cartesianas e elipsoidais com suas respectivas precisões). 
Neste trabalho realizou-se a construção de séries temporais utilizando o programa computacional desenvolvido, de forma a demostrar sua eficiência e potencialidade para aplicações às pesquisas. Foram efetuadas análises preliminares sobre o comportamento das séries de coordenadas cartesianas e suas tendências no período considerado, de forma a verificar/identificar descontinuidades. Uma análise estatística também foi efetuada entre as séries de dados linimétricos e as séries de altitudes geométricas, permitindo inferir sobre a influência da carga hidrológica na altitude geométrica, verificando o comportamento das séries e suas correlações.

\section{Geração de séries temporais de informações posicionais}

Com o intuito de automatizar a construção de séries temporais a partir de dados coletados por estações pertencentes à RBMC, desenvolveu-se um programa computacional fundamentado em linguagem de programação $\mathrm{C}$ orientada a objetos e Java. Este programa foi nomeado de RINEX EDITION e está disponível na página eletrônica do curso de Engenharia de Agrimensura e Cartográfica da Universidade Federal de Viçosa (www.eam.ufv.br).

O planejamento e desenvolvimento do programa foram executados visando oferecer ao usuário uma interface amigável e simples, abrangendo as quatro etapas necessárias para geração das séries, ou seja, o download, a edição, o pós-processamento dos dados e a extração de informações posicionais (coordenadas cartesianas $\mathrm{X}, \mathrm{Y}, \mathrm{Z}$; elipsoidais $\varphi, \lambda$, h e as precisões de ambas), minimizando consideravelmente o tempo demandado, reduzindo também possíveis erros ocasionados na etapa de edição realizada manualmente. O layout apresentado foi construído de forma a alocar cada etapa em uma aba, denominando-as respectivamente de RBMC, TEQC, PPP e EXPORTAR.

O fluxograma apresentado na Figura 1 ilustra o princípio de funcionamento de cada aba do programa, apresentados seguindo a ordem lógica de operação para a construção de uma série temporal. 


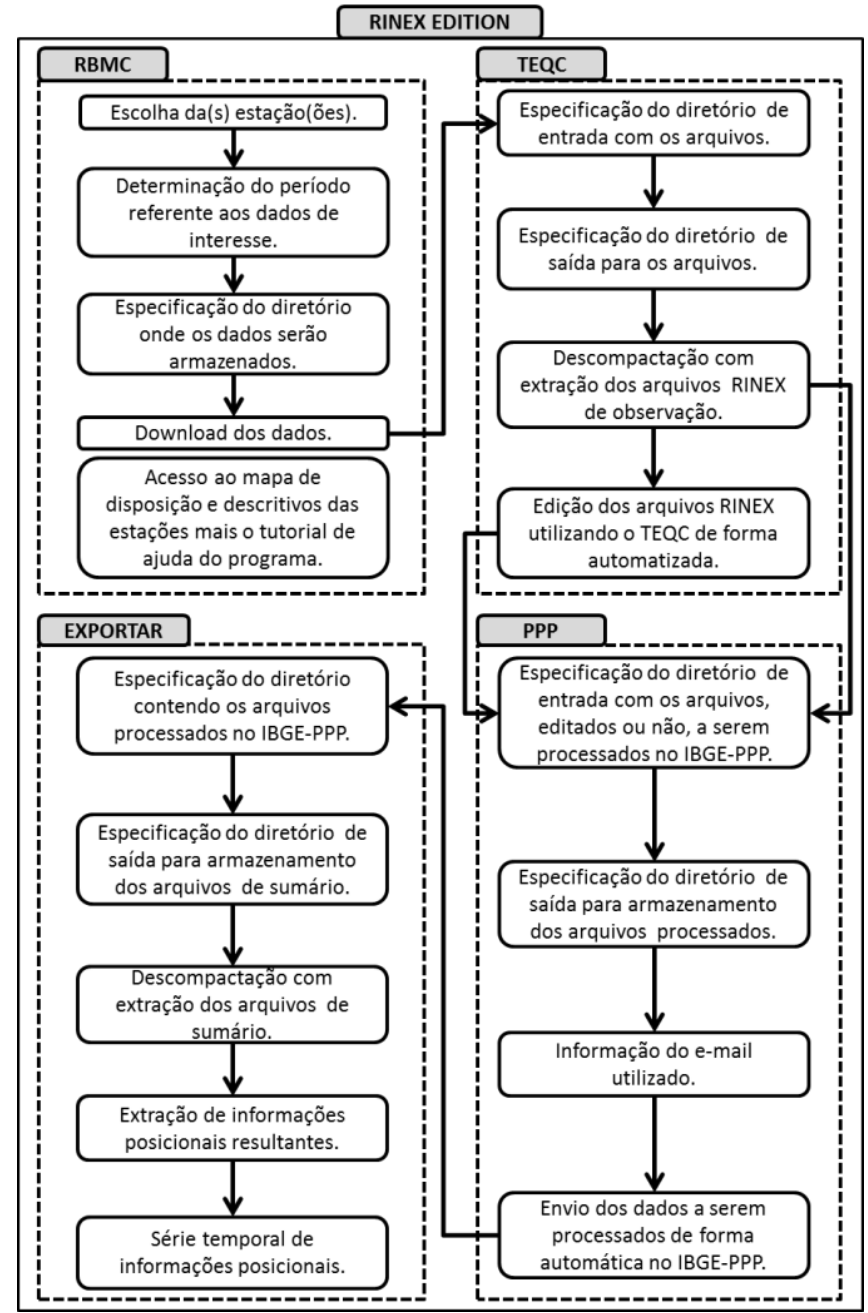

Figura 1: Fluxograma de funcionamento do RINEX EDITION.

O processo construtivo de uma série temporal se inicia na primeira aba (RBMC), em que é possível efetuar o download de dados de uma, ou mais estações pertencentes à RBMC. A ordem dos procedimentos necessários, bem como, de inserção de informações está disposta no fluxograma da Figura 1. Caso seja omitida alguma dessas informações será retornada ao usuário uma mensagem informando a inconsistência, não permitindo que se efetue o download. Somente depois de inseridas todas as informações solicitadas pelo programa se iniciará o download de forma automatizada. Para auxílio ao usuário são disponibilizados: o tutorial de ajuda do programa, um mapa da disposição das estações da RBMC e um link direto para os descritivos de cada estação. Cabe ressaltar que as datas devem pertencer ao mesmo ano. Em caso de um intervalo em anos diferentes, o usuário deverá efetuar o download em duas partes.

Com o download finalizado e os dados de interesse armazenados, tem-se início a descompactação e armazenamento dos arquivos de observação (aba TEQC), que podem ser editados ou não. A descompactação é efetuada utilizando os softwares Zip e UnZip, pertencentes ao projeto INFO-ZIP, disponibilizados de forma gratuita em <www.info-zip.org/>. A edição dos dados é executada de forma automática utilizando-se o software TEQC (Translation, Editing and Quality Check). O TEQC é disponibilizado de forma gratuita pela UNAVCO em <www.unavco.org/software/data-processing/teqc/teqc.html>.

Com o uso do TEQC, pode-se, por exemplo, retirar as observações referentes aos satélites da constelação GLONASS mantendo as observações do GPS, ou vice-versa; manter somente as 
observações da portadora L1 referentes às duas constelações de forma conjunta ou individual; redução do tempo de rastreio para intervalos específicos, tendo como referência o período total disponibilizado nos arquivos (0 a 24h).

Com os arquivos de observação preparados, inicia-se a terceira etapa (aba PPP), ou seja, o pósprocessamento. Esse procedimento constitui um dos empecilhos da geração das séries temporais que utilizam serviços de PPP online, pois demanda muito tempo. Pensando-se em sanar as dificuldades impostas construiu-se um aplicativo, utilizando a linguagem de programação Java de forma a estabelecer uma conexão direta ao serviço de pós-processamento online IBGE-PPP em modo estático. Este aplicativo funciona em conjunto com o RINEX EDITION e permite que o usuário envie seus dados ao serviço de processamento do IBGE recebendo e armazenando os arquivos processados no diretório especificado. A vantagem deste aplicativo evidencia-se quando considerado um grande volume de dados. Outra consideração importante é a possibilidade de envio de dados em paralelo, ou seja, com várias "janelas" funcionando ao mesmo tempo. A eficiência desse depende da largura da banda de internet disponível ao usuário.

A adoção do método PPP, em particular o serviço IBGE-PPP, se justifica por vários motivos, dentre eles: trata-se de uma aplicação disponibilizada por uma instituição brasileira, em língua portuguesa, que permite o posicionamento tanto no sistema global (ITRF) quanto no sistema oficial do país (SIRGAS 2000); a confiabilidade, acurácia e praticidade proporcionada por este serviço; facilidade de implementação no software desenvolvido, o que é difícil de ser feito com outros programas de processamento de dados no modo relativo (programas comerciais), dentre outros.

Moreira (2010) destaca que uma das grandes vantagens do modo PPP é a não necessidade de outras estações terrestres como referência, tornando as soluções independentes, o que pode ser considerado um grande beneficio em estudos sobre a determinação de variações locais das coordenadas da estação, pois evita propagação de erros e movimentos diferenciados entre estações, como acontece no método de dupla diferença.

É importante destacar que o IBGE-PPP, no âmbito de suas configurações utiliza: máscara de elevação ou ângulo de elevação (cut-off) de 10 (dez) graus, o que permite reduzir os efeitos do multicaminho; dados meteorológicos de superfície (temperatura, pressão e humidade relativa); modelos troposféricos (Davis, Hopfield e GMF); correção do erro de primeira ordem da ionosfera; órbitas precisas e correções para os relógios dos satélites (cabe salientar que informações de órbitas e relógios dos satélites são indispensáveis para o processamento e, na sua ausência, o processamento não ocorrerá); correção do centro de fase das antenas dos satélites e dos receptores (arquivos contendo os valores do desvio do centro de fase e suas variações para um conjunto de modelos de antenas, disponibilizados e atualizados constantemente pelo IGS e pelo NGS (National Geodetic Survey)); parâmetros de transformação entre os referenciais ITRF/SIRGAS2000; correção dos efeitos de carga oceânica, utilizando o modelo FES2004; correção do deslocamento de placas tectônicas, utilizando o modelo de velocidades VEMOS2009; correções de marés (polares e terrestres), aplicadas através dos parâmetros de orientação terrestre (ERP) oriundos das órbitas precisas, dentre outras. Para mais informações consultar IBGE (2013) e Costa et al. (2013).

A quarta e última etapa (aba EXPORTAR) consiste em solucionar outro problema associado à formação das séries e que também demanda tempo considerável: a extração de informações posicionais de dados pós-processados. Nesse caso, desenvolveu-se um algoritmo que permite a extração automática de tais informações a partir dos arquivos de sumário disponibilizados na terceira etapa, garantindo a otimização do processo. Tem-se assim uma série temporal de dados posicionais estimados na época de coleta dos dados. 


\section{Experimento, resultados e análises preliminares}

Para realização do experimento foram utilizados: Dados GNSS (GPS e GLONASS) em formato (RINEX) para as estações NAUS (Manaus - AM) e SAGA (São Gabriel da Cacheira - AM), referentes ao período considerado (01/01/2010 a 13/08/2015), disponibilizados pelo IBGE em sua página eletrônica; Programa computacional RINEX EDITION, versão 1.0; e dados fluviométricos (cotas linimétricas) referentes à estação fluviométrica MANAUS - ANA (código 14990000) e a estação SÃO GABRIEL DA CACHOEIRA - ANA (código 14320001), disponibilizados pela Agencia Nacional de Águas (ANA) através do seu banco de dados HidroWeb (ANA 2016; SNIRH 2016). A localização/disposição das estações pode ser verificada nas Figuras 2 e 3.

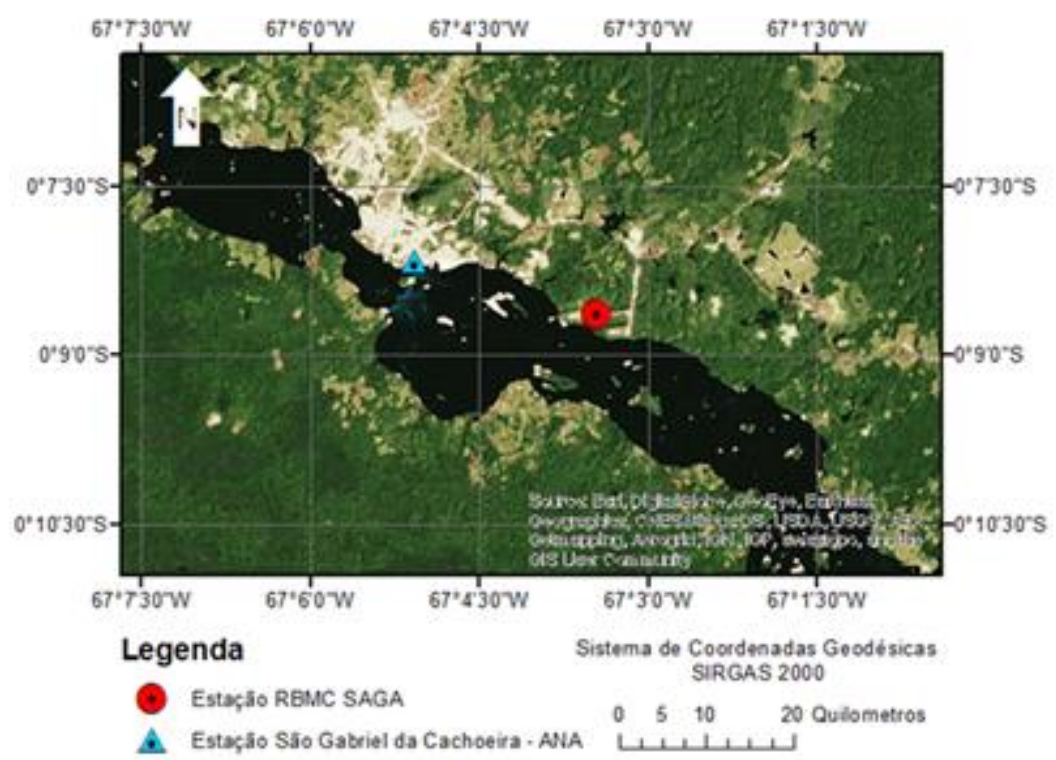

Figura 2: Disposição das estações São Gabriel da Cachoeira - ANA e RBMC SAGA.

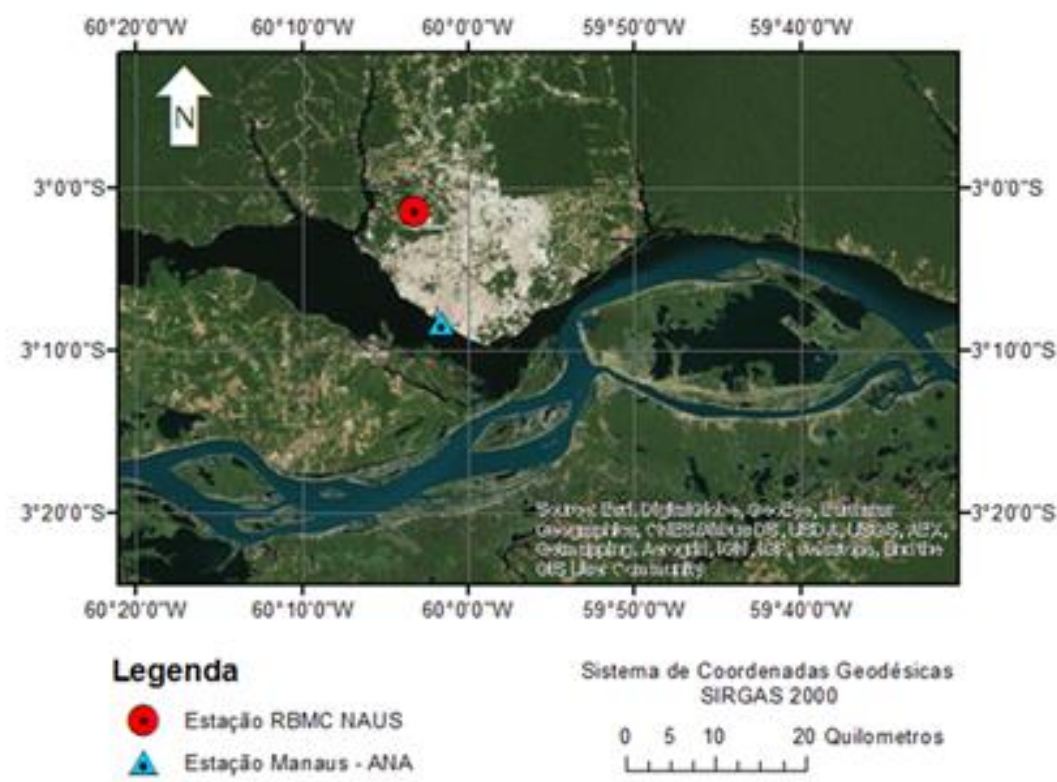

Figura 3: Localização das estações Manaus - ANA e RBMC NAUS. 
Com o programa supracitado foi possível gerar as séries temporais de dados posicionais para as estações NAUS e SAGA, pertencentes à RBMC, para o período iniciado no dia corrido de número 1 do ano de 2010 (01/01/2010) e finalizado no dia corrido de número 217 do ano de 2015 (05/08/2015) totalizando uma série temporal de aproximadamente cinco anos e meio (5,5 anos) para cada estação. Cabe salientar que o tempo destinado à construção dessas séries foi de 48 horas, utilizando uma conexão de internet de banda larga de 5MB, confirmando assim a eficiência do programa. Tal eficiência é justificada quando se compara o tempo demandado no procedimento manual (meses), com o tempo gasto no processo automatizado para a construção das séries posicionais aqui utilizadas. As séries de informações posicionais geradas com o programa computacional desenvolvido seguem o padrão apresentado na Figura 4.

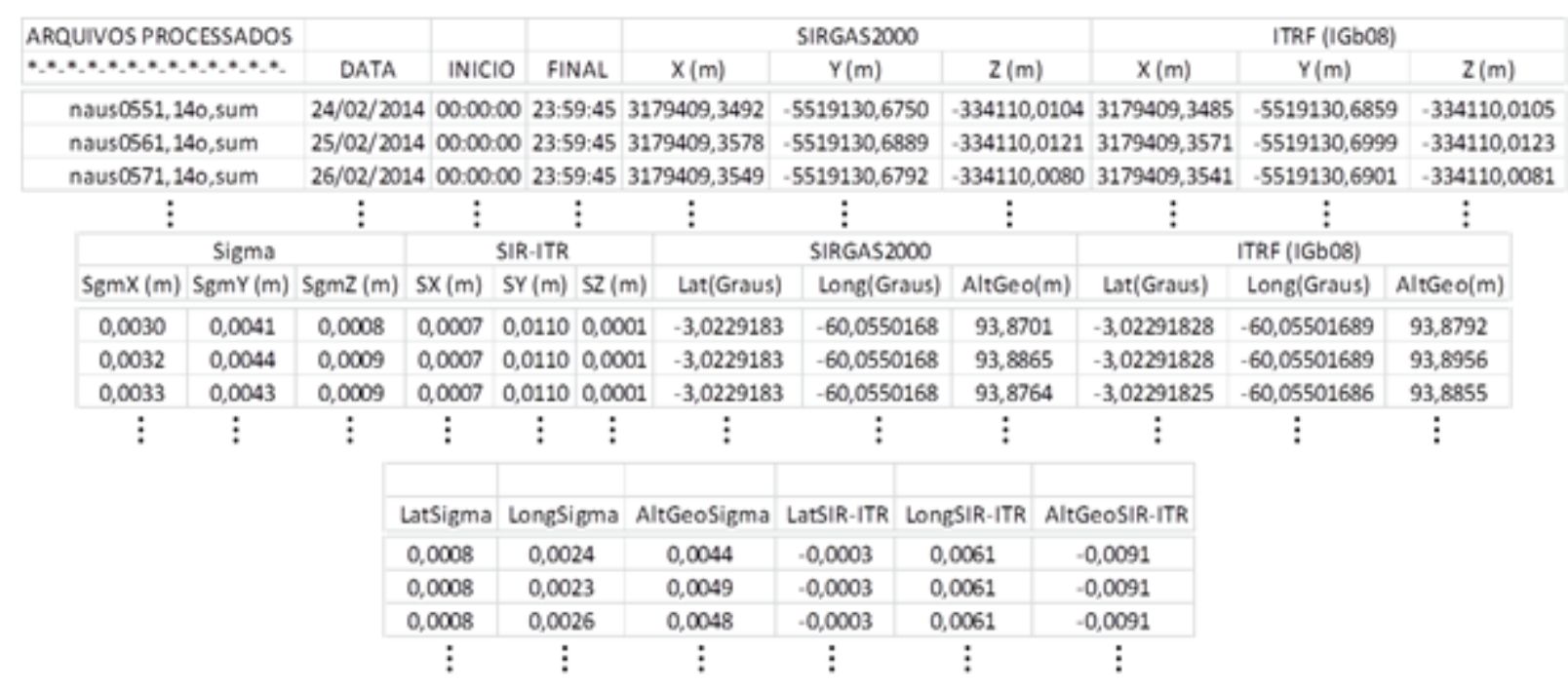

Figura 4: Série de informações posicionais geradas com o RINEX EDITION.

As coordenadas que compõem as séries são estimadas para a época de coleta dos dados. Vale ressaltar que, embora as informações posicionais (coordenadas cartesianas X, Y, Z; elipsoidais $\varphi$, $\lambda, \mathrm{h}$ e as precisões de ambas) resultantes do IBGE-PPP sejam também referenciadas ao SIRGAS2000, foram consideradas neste trabalho as coordenadas cartesianas, bem como as altitudes elipsoidais, referenciadas ao IGb08, o qual é alinhado ao ITRF2008. O ITRF2008 e o IGb08 são equivalentes ao nível global, no sentido de que eles compartilham a mesma origem, escala e orientação (Rebischung et al. 2012; Bruyninx et al. 2013).

Após a geração das séries temporais de coordenadas, foi verificada a presença de ruído aleatório, que se deve aos erros aleatórios do processamento de dados GNSS, o que poderia prejudicar os resultados. Nesse caso, é necessário que se aplique algum filtro às séries, de forma a minimizar tais ruídos. Com esse intuito, aplicou-se às séries um filtro de médias móveis simples, com uma janela de tamanho igual a 6 (seis), baseando-se nos resultados apresentados por Lima (2015), onde os erros aleatórios (ruídos) foram reduzidos satisfatoriamente. As séries posicionais filtradas podem ser observadas na Figura 5. Salienta-se que as análises apresentadas foram efetuadas sobre as séries posicionais filtradas.

É importante destacar que as séries temporais de coordenadas podem refletir, além dos efeitos da carga hidrológica, outros efeitos. Como evidenciado por Choy (2009), Costa et al. (2013), Abdelazeem e Çelik (2014) e MacLeod e Tétreault (2014), os principais efeitos de deslocamento associados às estações são: marés terrestres (Solid Earth Tides), marés polares (polar tides), 
cargas de pressão atmosférica (Atmospheric Pressure Loading) e carga oceânica (Ocean Loading). Tais efeitos, no caso do posicionamento PPP, devem ser considerados e, quando possível, modelados.

IBGE (2013) afirma que o serviço IBGE-PPP faz uso do programa CSRS-PPP (GPS Precise Point Positioning), desenvolvido pelo NRCan (Geodetic Survey Division of Natural Resources of Canada). Nesse contexto, diversas correções são implementadas neste programa e, consequentemente utilizadas pelo IBGE-PPP.

O CSRS-PPP, bem como o IBGE-PPP utiliza correções para marés terrestres, polares e carga oceânica, conforme especificações das convenções do IERS (International Earth Rotation Service). A correção de maré terrestre é uma das mais importantes realizadas no processamento PPP, tendo grande impacto (dependendo da frequência de processamento), nos resultados obtidos. As correções de marés (polares e terrestres) são aplicadas através dos parâmetros de orientação terrestre (ERP) oriundos das órbitas precisas. A correção da carga do oceano é efetuada no IBGE-PPP aplicando-se o modelo FES2004. Essas correções são utilizadas se a estação a ser processada estiver a uma distância de até $10 \mathrm{~km}$ de uma estação que possua valores de correção de carga oceânica. Mais informações sobre os modelos e correções citadas consultar IERS (2010), IBGE (2013) e Costa et al. (2013).

Segundo Moreira (2010), as convenções apresentadas em IERS (2010) não apresentam informações sobre a modelagem e/ou eliminação de efeitos de carga hidrológica. IERS (2010) disponibiliza uma secção (7.2) para a inserção da modelagem dos efeitos de deslocamento associados às mudanças de cargas ambientais (carga de pressão atmosférica e carga hidrológica), que está em desenvolvimento em diversos centros de estudos. Neste caso, recomenda-se não incluir tais modelagens em soluções operacionais que suportam produtos e serviços do IERS. Esta seção será atualizada à medida que modelos a serem adotados estiverem disponíveis.

Urquhart (2009) evidencia que os efeitos de carga da pressão atmosférica podem ser desprezíveis no PPP. Rosa (2008) justifica que tais efeitos afetam principalmente a componente vertical, apresentando amplitudes maiores em regiões de alta latitude, devido ao regime climático intenso e menores em regiões de latitude média e próximas ao oceano devido à resposta barométrica inversa dada pelo oceano. Como mostrado em IERS (2010), os efeitos da carga de pressão da atmosfera atingem cerca de $1,5 \mathrm{~mm}$ em amplitude, na componente vertical, considerando a região de estudo. Tais efeitos são 10 (dez) vezes menores para as componentes horizontais.

Nesse contexto, considerou-se que as séries temporais refletem, principalmente, os efeitos da carga hidrológica.

Podem-se observar na Figura 5 as séries temporais de componentes cartesianas geradas utilizando o RINEX EDITION, para as estações NAUS e SAGA. De forma a melhorar a visualização, efetuou-se a subtração das partes inteiras das coordenadas cartesianas, mantendo somente às diferenças centimétricas, facilitando a observação das variações temporais.

Aplicando-se a linha de tendência linear, observou-se que: as séries temporais das componentes $\mathrm{X}$ de ambas as estações apresentaram comportamento decrescente; a série da componente $\mathrm{Y}$ da estação NAUS apresentou tendência constante, caracterizando a estacionariedade da série, enquanto a série da mesma componente para a estação SAGA apresentou uma tendência decrescente para o período analisado; as séries das componentes $\mathrm{Z}$ de ambas as estações apresentaram comportamento crescente. Além disso, a série da componente $\mathrm{Z}$ referente à estação NAUS apresentou um deslocamento entre os anos de 2013 e 2014, o que não foi observado nas séries das demais componentes. Esse deslocamento coincide com o período da troca de antenas da estação, onde o modelo utilizado Zephyr GEODETIC (TRM41249. 00) foi removido em 09/04/2013 e alterado, nessa mesma data, para o modelo GNSS Choke Ring (TRM59800. 00), 
como pode ser observado no descritivo da estação, disponível em < ftp://geoftp.ibge.gov.br/RBMC/relatorio/>. No entanto, tal coincidência não pode ser especificada como a causa de tal deslocamento.

Dessa forma, infere-se que uma análise sobre as séries de coordenadas cartesianas é extremamente útil na avaliação do comportamento das séries e na busca de descontinuidades, possibilitando indicar possíveis erros nos dados da estação, o que mostra o RINEX EDITION como uma ferramenta excelente para auxiliar esses propósitos.

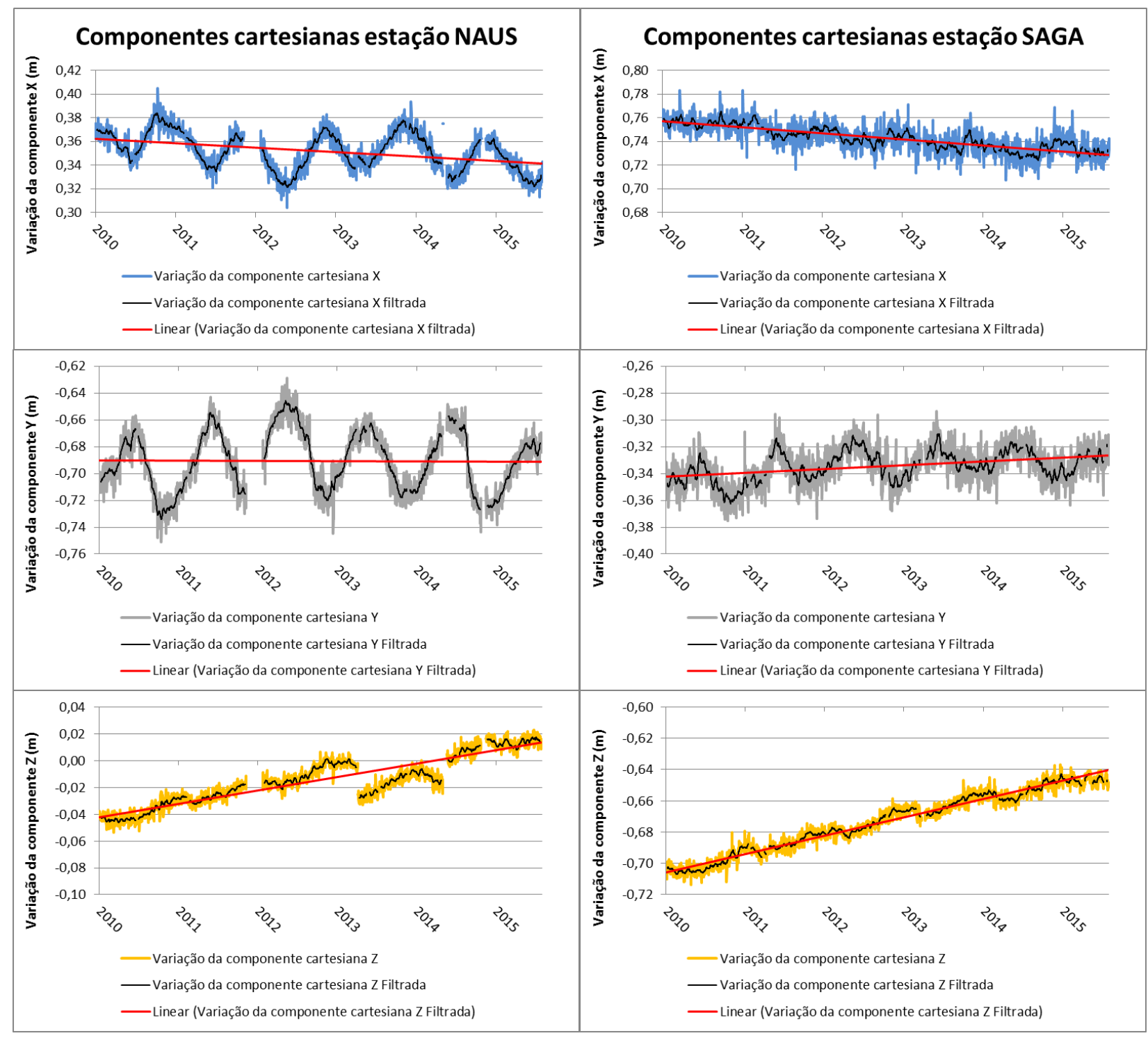

Figura 5: Séries temporais das componentes cartesianas $X, Y$ e $Z$ e suas linhas de tendência linear para as estações NAUS (à esquerda) e SAGA (à direita).

De forma a proceder à verificação do comportamento da altitude geométrica em função da carga hidrológica se fez necessário construir séries temporais de dados fluviométricos (cotas linimétricas) para posterior comparação e análise.

As séries de dados linimétricos foram construídas a partir de dados fluviométricos disponibilizados pela Agencia Nacional de Águas (ANA), em seu banco de dados HidroWeb, 
disponível em sua página eletrônica (ANA 2016; SNIRH 2016). Tais dados utilizados consistem nas médias diárias fornecidas para as estações MANAUS - ANA (código 14990000), estação fluviométrica do Porto de Manaus, situada nas proximidades da cidade de Manaus - AM e a estação SÃO GABRIEL DA CACHOEIRA - ANA (código 14320001), localizada nas proximidades do município de São Gabriel da Cachoeira - AM, no alto Rio Negro, como apresentado nas Figuras 2 e 3. Foram considerados como critérios de escolha das estações fluviométricas a disponibilidade de dados para o período coincidente ou aproximado com o período das discrepâncias calculadas. Outro critério importante se refere às distâncias das estações fluviométricas e da RBMC com relação aos rios. Nesse caso, as menores distâncias foram apresentadas pelas estações localizadas em São Gabriel da Cachoeira - AM. A primeira estação fluviométrica (Manaus) apresentou dados para o período de 01/01/2010 à 30/06/2014, enquanto a segunda (São Gabriel da Cachoeira) para o período de 01/01/2010 à 31/01/2015.

No que concerne à carga hidrológica, é importante destacar que esta se deve à variação de massa de água, causada pelo ciclo hidrológico, que envolve: a chuva, evaporação, infiltração e os escoamentos superficial e subterrâneo. O nível de água por sua vez, está associado, de forma significativa, ao escoamento superficial. No entanto, o volume escoado superficialmente é dependente, além do nível de água, da área da seção transversal do rio e da velocidade do curso de água. Acentua-se que foi feita uma simplificação, no âmbito da comparação das altitudes geométricas com o nível de água (cotas linimétricas), desconsiderando tais fatores. Destaca-se também que os valores apresentam uma boa correlação devido à boa correlação do nível de água com o escoamento e ao fato de o escoamento ser o principal causador da deformação crustal na região.

Efetuando uma análise comparativa entre a série de dados fluviométricos e a série de altitudes geométricas filtradas para a estação NAUS, apresentadas na Figura 6, infere-se que existe correlação inversa de $91,3 \%$, apresentada na Tabela 1.

Tabela 1: Correlações entre as séries de cotas linimétricas e as séries de altitudes geométricas.

\begin{tabular}{c|c|c|c|c}
\hline & $\begin{array}{c}\text { Cotas Linimétricas } \\
\text { Manaus }\end{array}$ & $\begin{array}{c}\text { Cotas Linimétricas } \\
\text { São Gab }\end{array}$ & $\begin{array}{c}\text { Altitudes } \\
\text { geométricas } \\
\text { filtradas NAUS }\end{array}$ & $\begin{array}{c}\text { Altitudes } \\
\text { geométricas } \\
\text { filtradas SAGA }\end{array}$ \\
\hline $\begin{array}{c}\text { Cotas Linimétricas } \\
\text { Manaus }\end{array}$ & $100,0 \%$ & $74,5 \%$ & $-91,3 \%$ & $-74,4 \%$ \\
\hline $\begin{array}{c}\text { Cotas Linimétricas } \\
\text { São Gab }\end{array}$ & $74,5 \%$ & $100,0 \%$ & $-74,1 \%$ & $-63,9 \%$ \\
\hline $\begin{array}{c}\text { Altitudes } \\
\text { geométricas } \\
\text { filtradas NAUS }\end{array}$ & $-91,3 \%$ & $-74,1 \%$ & $100,0 \%$ & $78,7 \%$ \\
\hline $\begin{array}{c}\text { Altitudes } \\
\text { geométricas } \\
\text { filtradas SAGA }\end{array}$ & $-74,4 \%$ & $-63,9 \%$ & $78,7 \%$ & $100,0 \%$ \\
\hline
\end{tabular}

Tal correlação também pode ser observada nos trabalhos desenvolvidos por Bevis et al. (2005), Moreira (2010) e Costa et al. (2012). O primeiro utilizou a correlação entre tais variáveis como um fator que possibilitasse evidenciar a influência da carga hidrológica na resposta elástica da 
crosta, pois seu intuito foi quantificar a deformação sazonal sofrida pela crosta nessa região, através da verificação das flutuações de massa no sistema do Rio Amazonas e da resposta elástica da Terra (módulo de compressão); o segundo teve como objetivo prioritário a geração de informações altimétricas a partir de receptores GNSS, com precisão centimétrica, de forma a possibilitar avaliações e melhorias na qualidade de dados de cotas fluviométricas obtidos por satélites orbitais e o terceiro efetuou a validação da avaliação pelo GRACE do acúmulo de água no subsolo da bacia amazônica com base em dados de linígrafos.

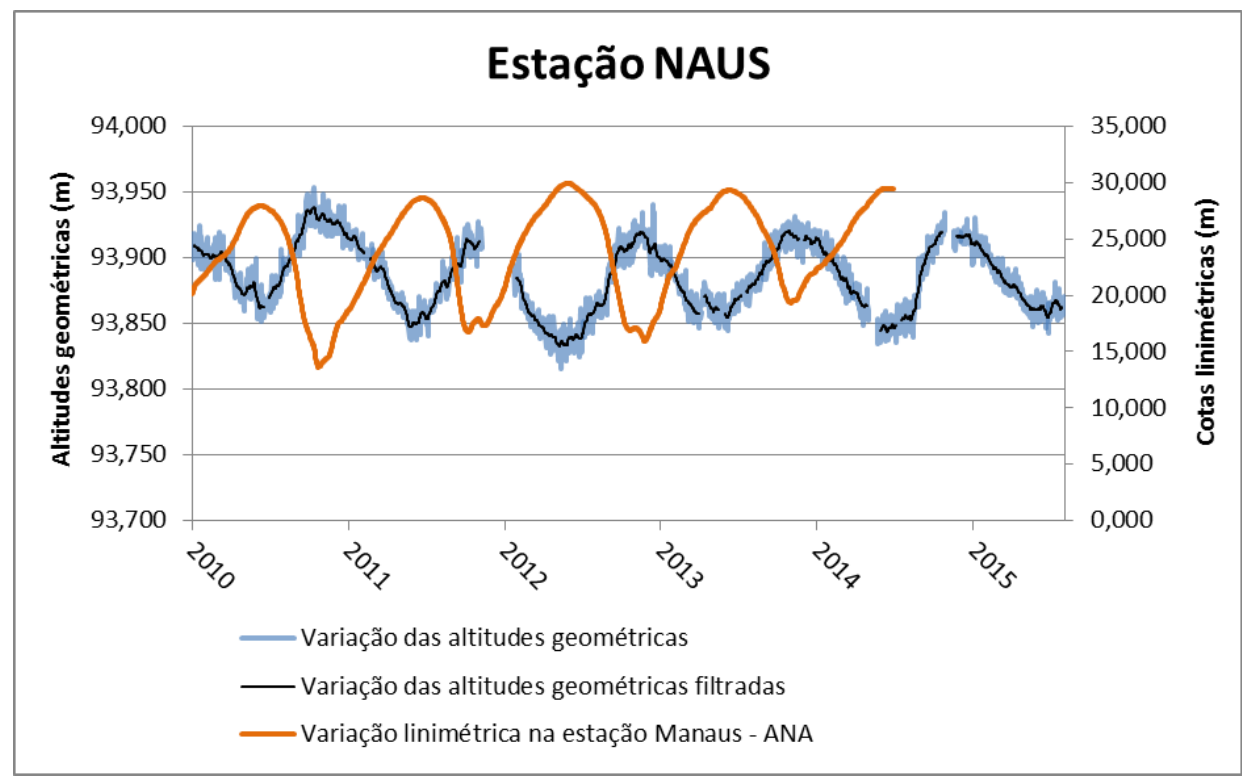

Figura 6: Comparativo entre a série de altitude geométrica da estação NAUS e a série linimétrica da estação MANAUS - ANA.

A série das altitudes geométricas para a estação SAGA apresentou um comportamento diferente quando comparada à série das altitudes da estação NAUS, apresentando maiores variações nos valores das soluções diárias, constituindo uma série mais ruidosa. Contudo, os ruídos apresentados nas séries talvez possam ser os mesmos, pois, como destaca Moreira (2010), a amplitude das variações de coordenada vertical encontradas em Manaus corresponde ao quádruplo das encontradas em São Gabriel da Cachoeira, o que pode contribuir para que os ruídos da série da estação SAGA sejam mais perceptíveis. Observa-se também que a série linimétrica referente à estação SÃO GABRIEL DA CACHOEIRA - ANA não apresentou comportamento bem definido, como apresentado na série de mesmo tipo para a estação MANAUS - ANA, o que pode ser visualizado na Figura 7. 


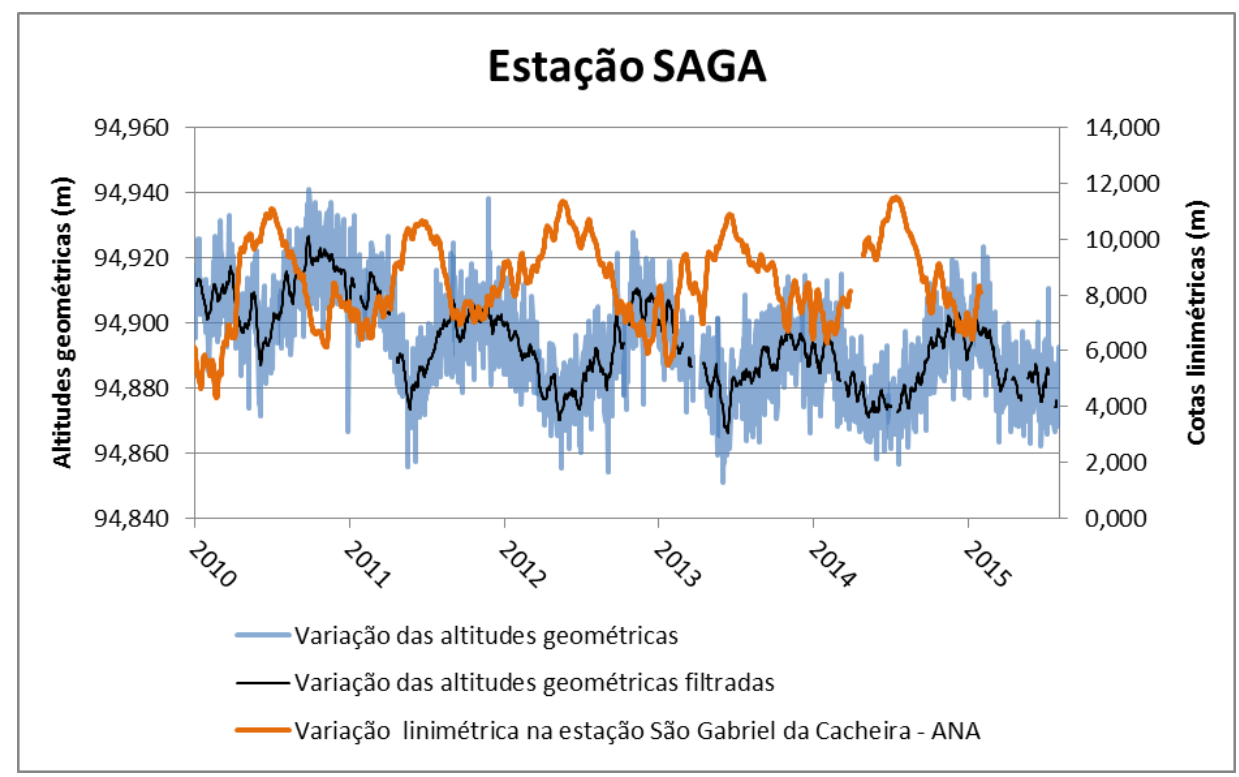

Figura 7: Comparação entre a série de altitude geométrica da estação SAGA e a série linimétrica da estação SÃO GABRIEL DA CACHOEIRA - ANA.

A estação SAGA apresentou, para o período analisado, uma correlação inversa de $63,9 \%$ entre as séries de altitudes geométricas e linimétricas, com o apresentado na Tabela 1. Quando comparada com a correlação apresentada pela estação NAUS, essa se mostra menor, fato justificável pela maior carga hidrológica encontrada em MANAUS - ANA. Evidencia-se que uma maior carga hidrológica contribui para melhorias na correlação, devido à amplitude do fenômeno ser maior e assim atenuar erros existentes na correlação.

Isso ocorre devido à estação NAUS se encontrar na zona de remanso, causada pelo encontro do Rio Negro com o Rio Solimões para a formação do Rio Amazonas, o que influencia diretamente na maior carga hidrológica local. A estação SAGA está localizada no alto Rio Negro, a noroeste de Manaus, a uma distância de aproximadamente $848,7 \mathrm{Km}$, não sofrendo influência significativa do Rio Amazonas. Bevis et al. (2005) constatou que os efeitos oriundos de carga hidrológica em Manaus constituem uma resposta elástica da crosta, e são mais significativos em um raio de até 200 quilômetros da estação. Costa et al. (2012) complementa que tal resposta pode ser ainda observada em bacias vizinhas, a uma distância de 1000 quilômetros de Manaus, mas com menor amplitude.

Cabe ressaltar que alguns locais de menor carga hidrológica podem apresentar melhor correlação devido a melhor representação da carga hidrológica pela variação linimétrica, ou mesmo uma melhor modelagem/impacto das outras correções que são aplicadas ao processamento GNSS, que permitem que o movimento das estações GNSS sejam apenas representados por variações da carga hidrológica. Esse fato pode ser observado em Moreira (2010), quando mostrou que as maiores correlações entre as séries altimétricas e de nível de água foram verificadas entre as estações de Porto Velho, e não às de Manaus. Cabe frisar que a amplitude das variações de coordenada vertical encontradas em Manaus correspondem ao dobro das encontradas em Porto Velho.

Destaca-se também que, mesmo com uma distância considerável entre as estações da RBMC e a diferença entre a vazão nas estações linimétricas, as estações RBMC apresentaram uma correlação direta de 78,7\% entre suas altitudes geométricas, conforme mostrado na Tabela 1. Esse valor se justifica, pois, grande parte do volume que passa em São Gabriel também passará em Manaus. Dessa forma, pode ser observada também na Tabela 1 uma correlação de 74,5\% entre as séries de cotas linimétricas. 


\section{Considerações finais, conclusão e recomendações}

Foram demonstradas neste trabalho as potencialidades do programa computacional RINEX EDITION para geração de séries temporais posicionais (coordenadas cartesianas $\mathrm{X}, \mathrm{Y}, \mathrm{Z}$; elipsoidais $\varphi, \lambda, \mathrm{h}$ e as precisões de ambas) de forma eficiente e eficaz. Com a utilização deste programa, geram-se inúmeras possibilidades de investigação sobre diversos fatores que afetam o PPP, considerando suas variações temporais. De acordo com os resultados obtidos foi possível verificar o efeito da carga hidrológica na variação da altitude geométrica. Salienta-se que este efeito não é considerado no PPP. Além disso, outros estudos podem ser efetuados, tais como: a análise da influência do ciclo solar, da ionosfera, troposfera, geodinâmicos, atmosféricos, carga hidrológica, etc.

Os resultados obtidos indicaram que o programa computacional desenvolvido se mostrou satisfatório, confirmando a utilidade do RINEX EDITION como uma ferramenta eficiente eficaz e de extrema importância para usuários que demandam o uso de séries temporais de dados posicionais utilizando dados GNSS coletados por estações pertencentes à RBMC.

As possibilidades de pesquisas que utilizam as séries temporais de informações posicionais são assim ampliadas, com séries construídas de forma rápida, com a possibilidade de edição ou não dos dados, e processamento automático utilizando o serviço IBGE-PPP. Por fim, a extração das informações assegura a extinção de erros associados a essa etapa, dispondo as informações posicionais de forma automática e organizada.

Observando as séries das variações das componentes cartesianas, verificou-se certa semelhança em seus comportamentos para ambas as estações, salvo para a componente $\mathrm{Z}$ da estação NAUS, que mostrou um deslocamento entre o ano de 2013 e 2014, o que não foi observado nas séries das demais componentes. Entretanto, não foi possível detectar a causa de tal deslocamento. Dessa forma, percebeu-se que uma análise sobre as variações das coordenadas cartesianas é extremamente útil, não somente para uma avaliação do comportamento das séries, mas também para a detecção de descontinuidades, o que mostrou o RINEX EDITION como uma ferramenta excelente para auxiliar nesse propósito.

Foi possível inferir que a ferramenta apresentada (RINEX EDITION) permite a geração de séries temporais posicionais para posterior análise, o que permitiu concluir, por exemplo, que a estação de Manaus sofre com maiores deformações em suas coordenadas verticais que a estação de São Gabriel da Cachoeira. Tal fato leva a manifestar maiores preocupações para correções destas deformações nessa região, de forma a garantir a qualidade dos levantamentos geodésicos.

Diante das análises preliminares apresentadas, propõe-se, como recomendação para trabalhos futuros, averiguar as causas da descontinuidade apresentada na série temporal da componente $\mathrm{Z}$ referente à estação NAUS. Além disso, observa-se em SIRGAS (2016) que as velocidades das estações de referência devem, preferencialmente, ser obtidos a partir da análise de séries temporais de posições obtidas por GNSS, que cubram um intervalo de tempo mínimo de dois anos. A partir de tal afirmação, recomenda-se aos usuários que buscarem realizar levantamentos geodésicos de precisão utilizando o PPP, que construam séries temporais posicionais para estações permanentes próximas a área de interesse, de forma a avaliar se movimentos da crosta terrestre, não modelados ou erroneamente modelados, prejudicam a precisão do levantamento realizado. O simples movimento da crosta pode ocasionar que um mesmo levantamento, dependendo da época de realização, possa apresentar diferença em suas coordenadas. 
Normalmente os levantamentos geodésicos visam estabelecer informações referidas a uma época comum e o fato da não correção destes movimentos, de certa forma, prejudicaria esse objetivo.

\section{AGRADECIMENTOS}

Os autores deste trabalho agradecem a CAPES pelo apoio em forma de bolsa de mestrado junto ao Programa de Pós-Graduação em Engenharia Civil - Informações Espaciais, da Universidade Federal de Viçosa e ao IBGE pela disponibilidade dos dados da RBMC e do serviço IBGE-PPP.

\section{REFERÊNCIAS BIBLIOGRÁFICAS}

Abdelazeem, M., e Çelik, R. N. 2014. "Accuracy and repeatability investigation of CSRS-PPP online processing servisse.” Coordinates 10 (5): 41-49. ISSN 0973-2136.

ANA (Agência Nacional de Águas). 2016. "HidroWeb". Acesso em 20 de março de 2016, http://hidroweb.ana.gov.br/default.asp

Box, G. E. P., Jenkins, G. M. e Reinsel, G. C. 2008. Time Series Analysis. New Jersey: John Wiley \& Sons.

Bruyninx, C., Altamimi, Z., Caporali, A., Kenyeres, A., Lidberg, M., Stangl, G. e Torres, J.A. 2013. "Guidelines for EUREF Densifications." IAG Sub-Commission for the European Reference Frame - EUREF. 5. Brussels, Belgium. Acesso em 20 de março de 2016, ftp://epncb.oma.be/pub/station/Guidelines_for_EUREF_Densifications.pdf

Bueno, R. F. 2007. "Monitoração, por GPS, de deslocamentos em estruturas com carga dinâmica." PhD diss., Universidade de São Paulo.

Bevis, M., Alsdorf, D., Kendrick, E., Fortes, L. P., Forsberg, B., Smalley Junior, R. e Becker, J. "Seasonal fluctuations in the mass of the Amazon River system and Earth's elastic response". Geophys. Res. Lett., 32 (16): 4. Acesso em 4 de junho de 2016, http://onlinelibrary.wiley.com/doi/10.1029/2005GL023491/abstract.

Choy, S. L. 2009. "An investigation into the accuracy of single frequency precise point positioning (PPP)." PhD diss., RMIT University.

Collischonn, C., Matsuoka, M. T., Lima, E. M., Waichel, F. S. e Camargo, P. O. 2014. "Correlação Do Posicionamento Por Ponto GNSS Com a Ionosfera E Com Indices de Atividade Solar No Periodo de 2002 a 2011." Boletim de Ciências Geodésicas 20 (4): 927-46. doi:10.1590/S1982-21702014000400051.

Costa, S. M. A., Matos, A. C. O. C. e Blitzkow, D. 2012. "Validation of the Land Water Storage from Gravity Recovery and Climate Experiment (GRACE) with Gauge Data in the Amazon Basin." Boletim de Ciências Geodésicas 18 (2): 262-81. doi:10.1590/S198221702012000200006.

Costa, S. M. A., Silva, A. L., Lima, M. A. A., Moura Júnior, N.J., Quirino, R. A. e Alonso, P. R. 2013. "Serviço online IBGE-PPP: avaliação dos 4 anos de serviço e perspectivas futuras." VIII Colóquio Brasileiro De Ciências Geodésicas. Curitiba, Brasil.

Grinter, T. e Roberts, C. 2011. "Precise Point Positioning: Where Are We Now ?" To Appear in Proceedings of the International Global Navigation Satellite Systems Society Symposium 
(IGNSS2011), Sydney, Australia.

Gu, G. e Wang, W. 2013. "Advantages of GNSS in Monitoring Crustal Deformation for Detection of Precursors to Strong Earthquakes." Positioning 04 (01): 11-19. doi:10.4236/pos.2013.41003.

IBGE (Instituto Brasileiro de Geografia e Estatística). 2013. "Manual do usuário aplicativo online IBGE-PPP Versão: Dezembro 2013". Editor: Coordenação de Geodésia - Diretoria de Geociências. Acesso em 07 de junho de 2016, http://www.ibge.gov.br/home/geociencias/geodesia/ppp/manual_ppp.pdf.

IERS (International Earth Rotation and Reference Systems Service). 2010. "IERS Conventions (2010)". Editor: Petit, G and Luzum, B. Acesso em 04 de junho de 2016. https://www.iers.org/IERS/EN/Publications/TechnicalNotes/tn36.html

Li, X. 2015. "Real-Time High-Rate GNSS Techniques for Earthquake Monitoring and Early Warning." PhD diss., Technischen Universität Berlin.

Lima, J. N. 2015. "A utilização de filtros digitais em séries temporais GNSS." Comunicação apresentada em VIII Conferência Nacional de Cartografia e Geodesia: VIII CNCG 2015. Lisboa, Portugal, 29 e 30 de outubro de 2015.

MacLeod, K e Tétreault, P. 2014. "NRCan Precise Point Positioning (PPP) Service ". In Civil GPS Service Interface Committee. Tampa, Estados Unidos.

Moreira, D. M. 2010. "Rede de referência altimétrica para avaliação da altimetria por satélites e estudos hidrológicos na região amazônica.” MS diss., Universidade Federal do Rio de Janeiro.

Ramos, M. P. 2015. “Análise Das Possibilidades de Transformação de Referencial E Atualização de Coordenadas No PPP.” MS diss., Universidade Federal de Viçosa.

Rebischung, P., Griffiths, J., Ray, J., Schmid, R., Collilieux, X. e Garayt, B. 2012. "IGS08: The IGS Realization of ITRF2008." Springer-Verlag 16: 483-94. doi:10.1007/s10291-011-0248-2.

Rosa, G. P. S. 2008. "Análise de séries temporais de coordenadas estimadas com GPS : uma proposta metodológica para eliminação de efeitos sazonais." MS diss., Universidade Estadual Paulista.

Shumway, R. H e Stoffer, D. S.. 2006. Time Series Analysisand Its Applications With R Examples. New York: Springer.

Silva, H. A e Monico, J. F. G. 2012. “Análise de Séries Temporais de Coordenadas GNSS.” In IV Simpósio Brasileiro de Ciências Geodésicas e Tecnologias da Geoinformação, 001-009. Recife, Brasil.

SIRGAS (Sistema de Referência Geocêntrico Para as Américas). 2016. "Modelo de velocidades para SIRGAS.” Acesso em 13 de junho de 2016. http://www.sirgas.org/index.php?id=54.

SNIRH (Sistema Nacional de Informações sobre Recursos Hídricos). 2016. "HidroWeb". Acesso em 20 de março de 2016, http://www.snirh.gov.br/hidroweb/.

Urquhart, L. 2009. "Atmospheric Pressure Loading and its Effects on Precise Point Positioning," Proceedings of the 22nd International Technical Meeting of The Satellite Division of the Institute of Navigation (ION GNSS 2009), Savannah, United States of America.

Recebido em 31 de dezembro de 2015.

Aceito em 08 de julho de 2016. 